\title{
Endovascular treatment of thoracoabdominal aortic aneurysms
}

\author{
Eric E. Roselli, MD, ${ }^{a}$ Roy K. Greenberg, MD, ${ }^{a, b}$ Kathryn Pfaff, BS, ${ }^{b}$ Catherine Francis, BS, ${ }^{b}$ Lars G. Svensson, MD, PhD, \\ and Bruce W. Lytle, MD
}

Earn CME credits at at http:// cme.ctsnetjournals.org

Ð Supplemental material is available online.
From the Departments of Thoracic and Cardiovascular Surgery ${ }^{\mathrm{a}}$ and Vascular Surgery, ${ }^{\mathrm{b}}$ The Cleveland Clinic Foundation, Cleveland, Ohio.

Roy Greenberg receives research support from Boston Scientific, Cook, Cordis Endovascular, Terumo-Vascutek, and W.L. Gore. He is a consultant for Boston Scientific and Cook and has intellectual property licensed to Cook. Bruce Lytle reports equity interest in Johnson \& Johnson. Eric Roselli reports consulting fees from Cook and lecture fees from Medtronic. Lars Svensson is an unpaid member of the multicenter steering committee for the FDA study of the Zenith Cook Stent Graft. All companies manufacture endovascular devices.

Read at the Eighty-sixth Annual Meeting of The American Association for Thoracic Surgery, Philadelphia, Pa, April 29-May 3, 2006

Received for publication May 10, 2006; revisions received Aug 8, 2006; accepted for publication Sept 26, 2006.

Address for reprints: Roy Greenberg, MD, Cleveland Clinic, 9500 Euclid Avenue, Desk S40, Cleveland, OH 44195 (E-mail: greenbr@ccf.org).

J Thorac Cardiovasc Surg 2007;133:1474-82 $0022-5223 / \$ 32.00$

Copyright $\odot 2007$ by The American Association for Thoracic Surgery

doi:10.1016/j.jtcvs.2006.09.118
Objective: To establish the safety and efficacy of endovascular repair of thoracoabdominal aortic aneurysms.

Methods: Between May 2004 and February 2006, patients with thoracoabdominal aneurysms considered high risk for conventional surgery were enrolled in a prospective trial to evaluate a novel endovascular grafting system. Devices were custom designed for each patient using high-resolution computed tomography. Patient data included mortality, morbidity, procedural details, and surrogate end points for endovascular repair. These were collected at hospital discharge and at 1, 6, and 12 months.

Results: Seventy-three patients underwent endovascular repair of thoracoabdominal aortic aneurysms for type I, II, or III $(n=28)$, or for type IV $(n=45)$ thoracoabdominal aneurysms. Mean aneurysm size was $7.1 \mathrm{~cm}$ (range $4.5-11.3 \mathrm{~cm}$ ). General anesthesia was used in $47 \%$ of patients and regional anesthesia in 53\%. There were no conversions to open surgery nor ruptures post-treatment. Technical success was achieved in $93 \%$ of patients (68/73). Thirty-day mortality was 5.5\% (4/73). Major perioperative complications occurred in $11(14 \%)$ patients and included paraplegia $(2.7 \%, 2 / 73)$, new onset of dialysis $(1.4 \%, 1 / 73)$, prolonged ventilator support $(6.8 \%, 5 / 73)$, myocardial infarction $(5.5 \%, 4 / 73)$, and minor hemorrhagic stroke $(1.4 \% ; 1 / 72)$. A majority of patients had no complications. Mean length of stay was 8.6 days. At follow-up, 6 deaths had occurred. There were no instances of stent migration nor aneurysmal growth.

Conclusions: Endovascular repair of aortic aneurysms involving the visceral segment in nonsurgical candidates is feasible. Known complications of repair are not eliminated, but morbidity and mortality appeared low relative to the high-risk population studied. Further refinement of device design, delivery technique, and patient selection is ongoing. Assessment of durability will require longer follow-up.

$\mathrm{S}$ tandard surgical approach to the treatment of thoracoabdominal aneurysms requires a demanding operation. Surgical outcomes are complicated by the presence of multiple comorbid conditions in these patients. Use of improved techniques has lowered the risk of complications and testifies to the effectiveness of treatment evolution and surgical experience in improving outcomes. However, risk for morbidity and mortality in this challenging patient population is still substantial. Large single-center experiences have reported mortality ranging from $7.4 \%$ to $17 \% .^{1-6}$ A recently published population-based study reported $20 \%$ perioperative mortality and only $69 \%$ survival at 12 months. ${ }^{7}$ Cardiac, neurologic, respiratory, and renal complications limit the number of patients eligible for surgery. All of these factors have encouraged the development of an alternative approach.

Endovascular repairs of straightforward infrarenal and thoracic aortic aneurysms have been favorably compared with surgical controls. ${ }^{\text {E1-E4 }}$ The application of this 


\section{Abbreviations and Acronyms \\ $\mathrm{CT}=$ computed tomography}

technology to segments of the aorta with visceral or brachiocephalic branches has required modifications of devices and procedures. Fenestrated devices have been developed to treat juxtarenal aneurysms, and the use of branched devices to treat aneurysms of the visceral and arch segments has been reported. ${ }^{8-13}$ We have explored a broadened indication for the use of endovascular procedures to treat aneurysms of the visceral segment. This article describes the techniques and reports the results of branched stent grafts to treat thoracoabdominal aortic aneurysms in select high-risk patients.

\section{Materials and Methods \\ Patients}

From May 2004 through February 2006, 73 patients with thoracoabdominal aortic aneurysms were evaluated in a prospective, nonrandomized fashion as part of a physician investigatorsponsored device exemption study, and the patients were treated with custom-designed branched endovascular devices. Written informed consent was obtained from all patients after full discussion of the purposes, risks, potential benefits, alternatives, need for follow-up studies, and investigational nature of the procedure. The study was approved by the Institutional Review Board. All patients were evaluated by surgeons who offer open and endovascular treatment alternatives. Preoperative assessment included functional stress tests and selective coronary angiography based on results, transthoracic echocardiography, pulmonary function testing, routine blood work, and physical exam.

Quantification of patients as poor candidates for conventional surgery is challenging, even for surgeons with extensive experience. The determination was made largely based upon the patient's age, aneurysm morphology, and comorbid factors present. ${ }^{1-7}$ All patients enrolled were considered high risk for open surgery after surgeons' assessment of all of these factors. Associated comorbidities are delineated in Table 1.

High-resolution computed tomography (CT) scans were acquired to evaluate the entire aorta and assessed on a 3-D imaging work station (Aquarius WS, Terarecon Inc, Mateo, Calif). Only patients treated for aneurysms that involved visceral aortic branches are reported.

\section{Device Construct}

The Zenith endograft (Cook Inc, Bloomington, Ind), previously studied for treatment of infrarenal abdominal aortic aneurysms, juxtarenal abdominal aortic aneurysms, and thoracic aneurysms, formed the basis of this device. ${ }^{\mathrm{E} 2, \mathrm{E} 3,8,10}$ Branches for the visceral segment were added to a tubular component that transcended the visceral segment in a manner similar to prior reports ${ }^{11,12}$ (Figure 1). Two types of branches were constructed depending upon the distance of the visceral ostia from the presumed location of the aortic prosthesis. The first involved a fenestration circumferentially rein-
TABLE 1. Patient and procedural characteristics

\begin{tabular}{|c|c|}
\hline Patient variables & $\mathrm{n}=73(\%)$ \\
\hline \multicolumn{2}{|l|}{ Demographic } \\
\hline Age $(y$, mean $\pm S D)$ & $75 \pm 8$ \\
\hline Male (no.) & $56(76)$ \\
\hline \multicolumn{2}{|l|}{ Aneurysm characteristics } \\
\hline Size $(\mathrm{mm}$, mean $\pm \mathrm{SD})$ & $70.7 \pm 15.1$ \\
\hline Type I/II/III & $77.3 \pm 16.8$ \\
\hline Type IV & $66.5 \pm 12.5$ \\
\hline \multicolumn{2}{|l|}{ Distribution (no.) } \\
\hline Type I/II/III & $28(38.4)$ \\
\hline Type IV & $45(61.7)$ \\
\hline \multicolumn{2}{|l|}{ Comorbidities (no.) } \\
\hline HTN & $49(67)$ \\
\hline DM & $13(18)$ \\
\hline CAD & $44(60)$ \\
\hline LVEF $<35 \%$ & $19(26)$ \\
\hline $\mathrm{CHF}$ & $21(29)$ \\
\hline $\mathrm{h} / \mathrm{o} \mathrm{Ml}$ & $25(34)$ \\
\hline h/o coronary revascularization & $18 \mathrm{CABG}, 11 \mathrm{PCl}(40)$ \\
\hline AICD & $11(15)$ \\
\hline COPD & $34(47)$ \\
\hline Home $\mathrm{O}_{2}$ requirement & $14(19)$ \\
\hline Baseline creatinine $\geq 1.5 \mathrm{mg} / \mathrm{dL}$ & $19(26)$ \\
\hline Serum creatinine $(\mathrm{mg} / \mathrm{dL}$, mean $\pm \mathrm{SD})$ & $1.4 \pm 0.9$ \\
\hline Cerebrovascular disease & $8(11)$ \\
\hline Prior aortic surgery & $21(29)$ \\
\hline Perioperative details & $\mathrm{n}=73(\%)$ \\
\hline Technical success (no.) & $68(93)$ \\
\hline $\begin{array}{l}\text { Angiographic evidence of aneurysm } \\
\text { exclusion }\end{array}$ & $73(100)$ \\
\hline Complete branch revascularization & $69(95)$ \\
\hline \multicolumn{2}{|l|}{ Anesthesia (no.) } \\
\hline Regional & $39(53)$ \\
\hline General & $34(47)$ \\
\hline \multicolumn{2}{|l|}{ Time (min, mean \pm SD) } \\
\hline Total & $320 \pm 106$ \\
\hline Fluoroscopy & $73.9 \pm 38.3$ \\
\hline Contrast volume $(\mathrm{mL}$, mean $\pm \mathrm{SD}$ ) & $210 \pm 99$ \\
\hline \multicolumn{2}{|l|}{ Access (no.) } \\
\hline Common iliac conduit & $7(10)$ \\
\hline \multicolumn{2}{|l|}{ Length of stay (days, mean \pm SD) } \\
\hline ICU & $3.2 \pm 5.2$ \\
\hline Total & $8.6 \pm 9.8$ \\
\hline
\end{tabular}

$H T N$, Hypertension; DM, diabetes mellitus; $C A D$, coronary artery disease; $\angle V E F$, left ventricular ejection fraction; $C H F$, chronic heart failure; $M I$, myocardial infarction; $A I C D$, automated internal cardiac defibrillator; $C O P D$, chronic obstructive pulmonary disease requiring inhaler therapy; $C A B G$, coronary artery bypass grafting; $P C l$, percutaneous coronary intervention; ICU, intensive care unit.

forced with a nitinol ring (reinforced fenestrated design). This was mated with a balloon-expandable stent graft partially extending within the aortic stent-graft lumen. The aortic segment of the balloon-expandable stent graft was then flared using sequentially larger balloons (12-mm, then a compliant 32-mm balloon) to abut the stent graft to the aortic graft, achieving a seal around the nitinol 


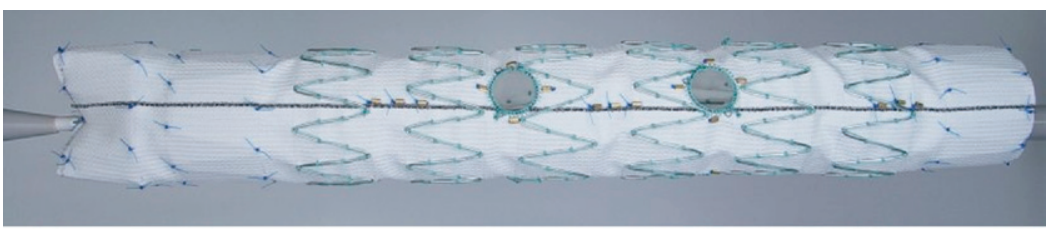

A

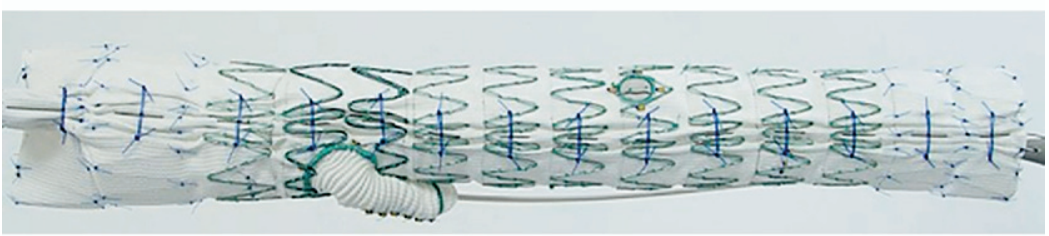

Figure 1. Main body device tethered to delivery system, demonstrating branch fenestrations for the (A) celiac and superior mesenteric arteries and (B) helical branch in antegrade orientation for the celiac artery (posterior view) and a reinforced fenestration for the left renal artery. Also note the posterior tethering sutures in (B). ring. The second branch type, termed a directional branch, consisted of an 8-mm polyester graft sewn to the aortic prosthesis above the target vessel. The branch was wrapped in a helical fashion external to the aortic prosthesis, oriented in either an antegrade or retrograde fashion, and terminated proximal to the target vessel. Directional branches provide long regions $(2 \mathrm{~cm})$ of overlap, allowing for the mating of a self-expanding stent graft (Fluency, Bard Inc, Tempe, Ariz) sized to the visceral vessel. A given device may have incorporated both types of branches based on the patient's anatomy.

\section{Device Design}

All devices were modular in design. The proximal and distal extent of aneurysmal disease, fixation and sealing zones, luminal diameters, and precise relationship between the visceral branches were determined using 3-D imaging. Sealing regions were oversized by $10 \%$ to $20 \%$. Device lengths were calculated from centerline of flow reconstructions with the objective of covering the minimum amount of aorta necessary while preserving internal iliac circulation. Additional internal iliac branches were used when needed. ${ }^{12}$

\section{Procedures}

Procedures included bilateral femoral artery exposure, anticoagulation to maintain activated clotting times greater than $300 \mathrm{sec}-$ onds, and selective exposure of brachial access sites. The primary device was delivered over a stiff wire that terminated in the ascending aorta. Each branch component was introduced through the contralateral femoral or brachial artery.

Longitudinal positioning of the aortic component was assisted by small injections of contrast above the celiac artery or by selective access of branch arteries to mark their positions. The device was then unsheathed. A posterior tethering wire partially constrained the graft, allowing fine positioning adjustments during selective cannulation of each visceral branch from within the aortic prosthesis.

Antegrade helical branches were selectively accessed with a preloaded wire and catheter. Retrograde helical branches and reinforced fenestrations were catheterized from femoral access points. Curved 7F or $8 \mathrm{~F}$ sheaths were placed into respective branches.

After access into each visceral vessel through the intended branch, the aortic component was completely expanded by removal of the posterior tethering wire. Release of the proximal fixation component allowed barbs to engage within the aortic wall. Balloon-expandable stent grafts (reinforced fenestrated branches) that were 17 to $38 \mathrm{~mm}$ in length (Jomed, Abbott Labs, Abbott Park, Ill) or self-expanding stent grafts (helical branches) that were $60 \mathrm{~mm}$ in length (Fluency, Bard, Tempe, Ariz) were delivered. Balloon-expandable stent grafts were flared. The aortic delivery system was removed and proximal thoracic, distal bifurcated iliac, or internal iliac components were added as required.

\section{Perioperative Patient Management}

Regional anesthesia was preferentially used in patients with significant comorbid pulmonary disease (Table 1). Patients were usually followed in an intensive care unit for a minimum of 12 hours ( $\mathrm{n}=$ 53). Spinal drainage was selectively employed depending on extent of aortic coverage (type I, II, and III aneurysms) or in the setting of prior aortic repair $(n=37)$. Drainage was continued for 72 hours or until CT scan confirmed aneurysm exclusion. Hydration and N-acetyl cysteine were administered before and after procedures for patients with chronic renal insufficiency. Other than aspirin, no anticoagulation was prescribed as a result of the aortic procedure.

\section{Follow-up}

Imaging and clinical evaluations occurred at 1, 6, and 12 months postoperatively and annually thereafter. Mortality data were available for all patients, and $92 \%$ of patients were compliant with clinical and imaging follow-up. Studies included serum creatinine and blood urea nitrogen, CT, visceral duplex ultrasonography, and plain chest and abdominal radiographs. Image evaluation was conducted on a work station using 3-D techniques (Figure 2).

\section{Outcome Definitions, Database, and Statistical Management}

The main objectives of therapy were to exclude the aneurysm and revascularize all associated branches during a single procedure. Technical success was defined by prosthesis implantation with patency of all intended branches, absence of angiographic evidence of type I or III endoleaks, and survival through 24 hours. Other end points included acute and late mortality; neurologic, cardiovascular, respiratory, and renal complications (Appendix E1); and radiographic outcomes consistent with reporting standards for endovascular ${ }^{\mathrm{E} 6}$ aneurysm repair. 


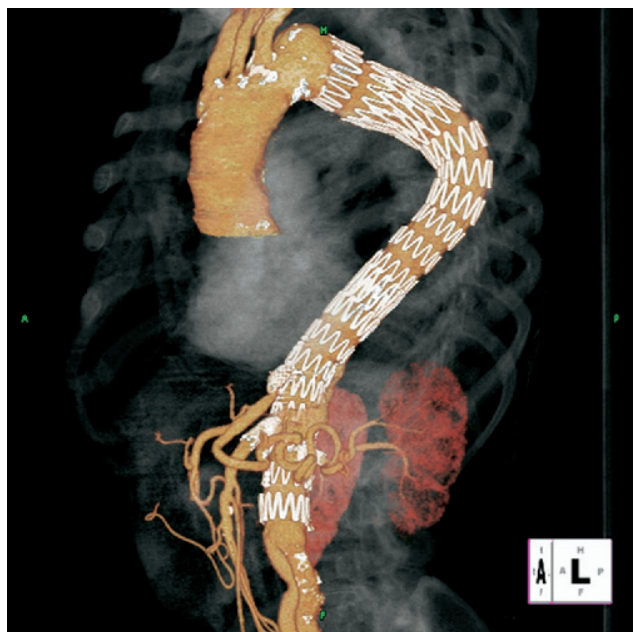

A

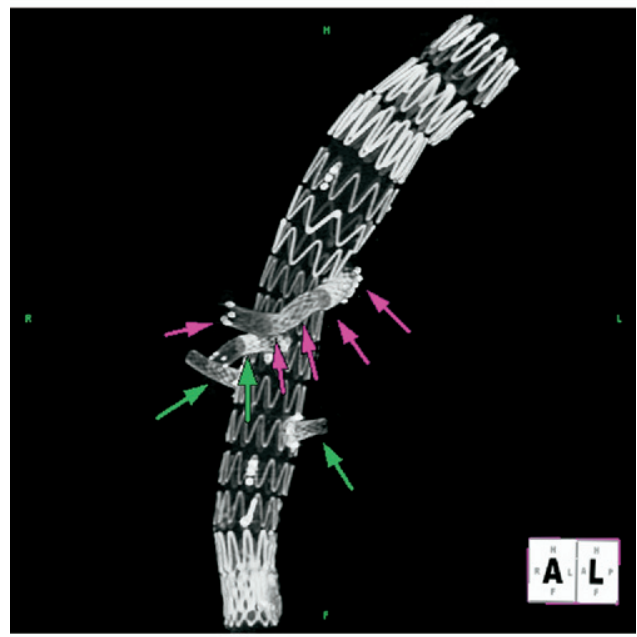

B

Figure 2. Completion CT scans post procedure. (A) Arterial contrast phase reconstruction of a type II thoracoabdominal aneurysm after repair with a 4-branch reinforced fenestrated device. (B) Noncontrast images reconstructed to demonstrate the in vivo appearance of a stent graft used to treat a type III thoracoabdominal aneurysm using composite device design with a helical celiac branch (purple arrows) and 3 reinforced fenestrated branches (green arrows).

Data were entered into an Oracle database. Mortality was assessed using life table analyses. Branch patency during follow-up studies was defined by contrast CT or duplex ultrasound, and device migration was defined according to recent modifications. ${ }^{14}$

\section{Results}

Technical Success

Technical success was achieved in 68 (93\%) patients. There were no conversions to open repair. Five technical failures included a single death within 24 hours, and 4 patients in whom we were unable to access a single branch artery. Three of these 4 patients underwent a second procedure to gain access into the failed branch during the same hospitalization in an effort to limit the initial contrast dose. A celiac artery was successfully accessed and stent grafted, and none of 3 renal arteries were salvaged.

\section{Mortality}

Probability of freedom from all-cause mortality $(94 \%, 85 \%$, and $81 \%$ ) and aneurysm-related mortality (94\%, 89\%, and $89 \%$ ) at 1, 6, and 12 months, respectively, is shown in Figure 3.

Acute mortality. Four patients died within 30 days, 2 prior to discharge. One died from a myocardial infarction within 24 hours of completing the procedure (which included a common iliac endarterectomy and iliofemoral bypass for device delivery), and the other died on day 7 from worsening biventricular failure. The other 2 acute mortalities followed discharge: 1 from mesenteric ischemia and 1 from renal failure.

Late mortality. Six patients died more than 30 days after surgery. Two patients expired at extended care facilities from complications related to spinal cord injuries. One died at an outside hospital due to an upper gastrointestinal bleed 4 days following embolization of a type II endoleak from the inferior mesenteric artery. A fourth late death resulted from sepsis 6 months after the procedure. The fifth patient died on day 167 from an acute myocardial infarction documented by autopsy, which also provided the opportunity for device explantation and analysis. And a sixth death occurred on postoperative day 312 as a result of cardiac arrhythmia and renal failure.

\section{Morbidity}

Thirty-seven $(52 \%)$ patients had no complications and were discharged after a mean of $4.9 \pm 3.0$ days. Death or major complications occurred in 15 patients (Table E1).

Neurologic complications. One patient had a minor hemorrhagic stroke from which he recovered during hospitalization. Two patients had spinal cord injury: one developed paraplegia and the other paraparesis. Both were delayed (3 days postprocedure), and both patients ultimately died at extended-care facilities. These patients had compromised internal iliac perfusion before the endovascular repairs, and both had prophylactic drainage of cerebrospinal fluid in place at the time of the initial procedure.

Cardiovascular and respiratory complications. None of the 10 patients with ischemia on preoperative testing had cardiac events. Four patients had perioperative myocardial infarctions. Overall, 5 patients $(6.8 \%)$ required prolonged intubation. Two of these who underwent tracheostomy are also the patients who had spinal cord injuries. 


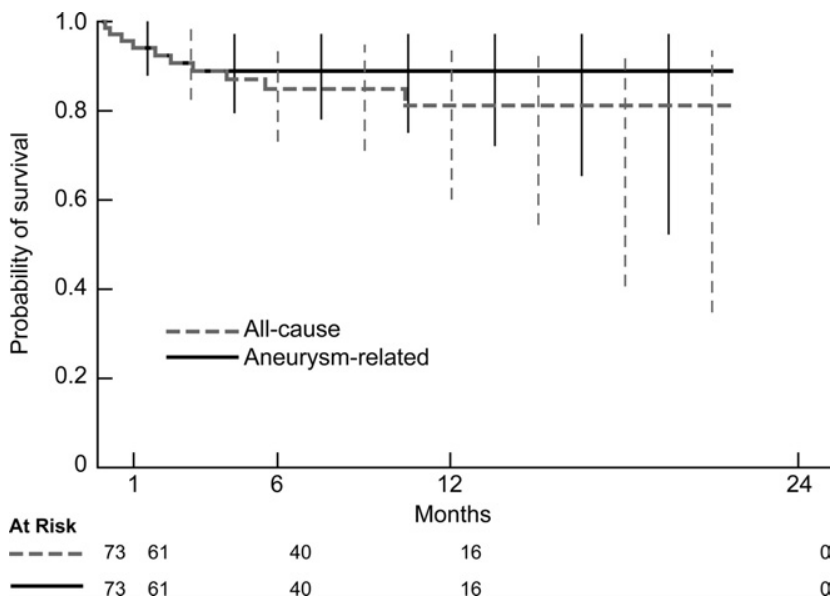

Figure 3. Kaplan-Meier estimate of the survival function for freedom from all-cause mortality (black) and aneurysm-related (b/ue) mortality, with pointwise $95 \%$ confidence limits.

Renal complications. Sustained elevations of serum creatinine greater than $30 \%$ over baseline levels were noted in only $11 \%(6 / 53)$ of patients at 1 -month follow-up. Two of these 6 patients had evidence of renal insufficiency preoperatively. One, who had paraparesis, required hemodialysis and eventually died. The other developed postoperative renal artery stenosis, which was treated with repeat stenting, and his serum creatinine has remained stable at $3.1 \mathrm{mg} / \mathrm{dL}$. Creatinine elevations in the other 4 patients have remained stable between 1.4 and $2.0 \mathrm{mg} / \mathrm{dL}$ at 6-month $(\mathrm{n}=2)$ and 12-month $(\mathrm{n}=2)$ follow-up.

Three patients had failed access into a single renal artery. At follow-up, 1 of these individuals had a reduced serum creatinine attributable to successful treatment of a tight stenosis in his dominant kidney. The second patient had a rise in serum creatinine, which has remained stable at 2.7 $\mathrm{mg} / \mathrm{dL}$. The third refused hemodialysis and died on postoperative day 30 .

\section{Radiographic Complications}

Endoleaks and sac morphology. Overall incidence of endoleaks was $11 \%$ before hospital discharge. Incidence and categorization of endoleaks are depicted in Figure 4. All type I $(n=3)$ endoleaks and all but 1 type III $(n=5)$ endoleaks were treated with secondary interventions. The single exception, a type III endoleak from the joint between the visceral component and bifurcated iliac component, resolved within 1 month. Of the 7 patients with type II endoleaks, 2 underwent treatment with glue embolization, 2 resolved by 1-month follow up, and the remaining 2 will be followed.

There have been no known aneurysm ruptures and no cases of sac enlargement over the follow-up period. Within

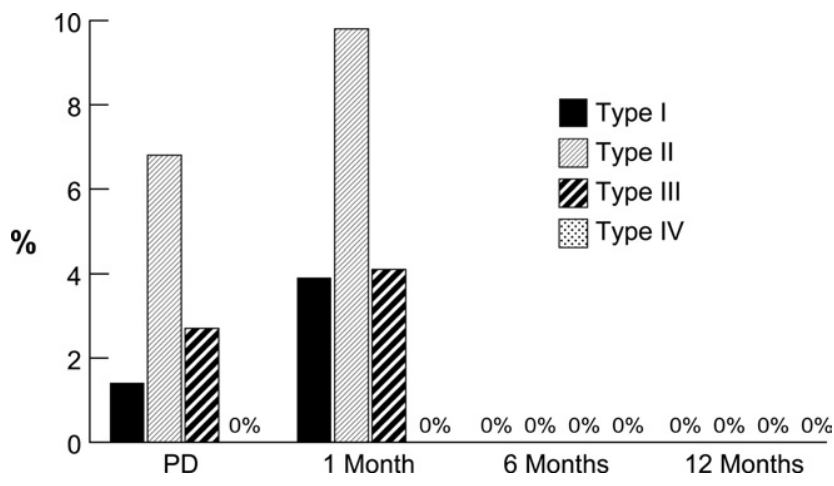

Figure 4. Number and classification of endoleaks by type at each interval of follow up. $P D$, Predischarge.

6 months, sac size decreased in $44 \%$ of patients, and at 12 months, $100 \%$ of eligible patients had documented sac shrinkage of greater than $5 \mathrm{~mm}$.

Secondary interventions. All secondary interventions were elective, and most occurred within the first month. Interventions for type I or III endoleaks occurred either before discharge $(n=3)$ or at 1-month follow-up $(n=4)$. Additional secondary procedures included groin complications $(n=2)$, internal iliac artery stenosis $(n=2)$, thrombosed iliac graft limb $(\mathrm{n}=1)$, and a renal stent fracture $(\mathrm{n}=1)$. Freedom from secondary interventions, by life table analysis, at 1, 6, and 12 months was $87 \%, 74 \%$, and $71 \%$, respectively.

Device integrity and branch patency. There have been no component separations, barb fractures, or device migrations. A single stent fracture was identified within a renal stent graft (Jomed) placed into a reinforced fenestration. This was not associated with any clinical event or endoleak and was treated with supplemental renal stent-graft placement. All renal and visceral branch vessels patent at hospital discharge remain patent at late follow-up imaging (Table E1).

\section{Discussion}

These patients were selected for endovascular versus open repair because of serious comorbid pulmonary, cardiovascular, or renal conditions, complex aortic morphology, and a high risk for aneurysm rupture. With these characteristics in mind, the perioperative and 12-month mortality rates of $5.5 \%$ and $19 \%$, respectively, compares favorably to previously published reports. ${ }^{1-7}$

Direct comparison between historic results of open surgery and the current study, however, is not accurate given that the patients in this series were deemed excessively high-risk candidates for an open repair. When compared with patients in other series, our patients were generally older; had more coronary artery disease, obstructive pulmonary disease, and chronic renal insufficiency; and had more 
frequently undergone prior abdominal aortic repair. ${ }^{1-7}$ Furthermore, these data were collected prospectively under an intensive clinical and imaging follow-up protocol that allowed for accurate charting of complications, a more reliable process than retrospective review.

An alternative hybrid approach to treating these patients combining open surgical extra-anatomic bypass with endovascular coverage of the visceral segment has been proposed as a potentially lower-risk procedure for these difficult-to-treat patients. In a recent review of our own experience with this technique, we found a $23 \%$ mortality rate and the need for additional open surgical procedures necessary in 6/13 patients. ${ }^{15}$ These results mirror those of others and have led us to reserve this approach to those with no other reasonable options, such as emergency cases or those with anatomy that would preclude branched device design (excessive tortuosity or compact branch vessel origins).

The decision to treat these patients with any invasive therapy must be weighed against the risk of aneurysm rupture. With the mean size exceeding $7 \mathrm{~cm}$, it can be estimated from the data provided by Elefteriades ${ }^{16}$ that the lifetime risk of complications in these patients exceeds $43 \%$. There have been no known ruptures or evidence of aneurysm growth after endovascular treatment in this series. Even with short-term follow-up, we have documented a decrease in aneurysm size in many patients, lending credence to the hypothesis that such a repair reverses the natural history of disease.

It is clear from these data that endografting has not eliminated the complications of spinal cord ischemia, renal dysfunction, and myocardial infarction associated with the treatment of thoracoabdominal aortic aneurysms; however, the occurrence of such events compares favorably with results from open surgical treatment. The paucity of pulmonary complications was particularly favorable. Ability to perform this procedure using regional anesthesia allowed for the treatment of patients with severe lung disease, a factor previously shown to be predictive of poor outcome. ${ }^{1,3,17}$ Avoidance of aortic crossclamping, obligatory end organ ischemia, and excessive fluid loss also potentially contributed to reduce risk.

Risk of spinal cord ischemia was relatively low in this series $(2.7 \%$ overall, $7.1 \%$ if all type IV thoracoabdominal aneurysms were excluded), despite the inability to reimplant intercostal arteries. This parallels the low incidence of spinal cord complications noted after endovascular repair of thoracic aneurysms and after extra-anatomic bypasses combined with total aortic coverage. ${ }^{10,11,18}$ However, the consequences of this complication are devastating, exemplified by the 2 patients in this series who ultimately died after a complicated postoperative course. Both patients had compromised internal iliac perfusion and extensive aortic coverage. Increased risk of spinal cord ischemia is an unavoid- able situation when treating extensive continuous aneurysmal disease. This risk in endovascular repairs seems to be more attributable to anatomic factors related to the morphology of the disease than to the physiologic consequences inherent to aortic clamping and open surgery. Therefore, cerebrospinal fluid drainage is employed in all patients at anatomic risk for spinal cord injury: those with type I to III aneurysms and those who have had prior aortic surgery. Similar concerns have been raised about diminishing left subclavian and consequently antegrade vertebral artery flow with proximal placement of thoracic endovascular devices. ${ }^{10,19}$ These observations have caused us to temper our enthusiasm for extensive endovascular repair in the setting of compromised pelvic or subclavian perfusion. Subsequently, such patients have either been turned down for a repair, undergone initial carotid subclavian bypass procedures $(\mathrm{n}=1)$, or had internal iliac branch device placement $(n=3)$ to maintain antegrade perfusion to these important collateral vascular beds. No patients had coverage of the left subclavian artery without a bypass procedure.

Although the stringent definition likely overestimates renal dysfunction in this series compared with other published series, the conclusions are similar: patients with thoracoabdominal aneurysms are at risk for renal impairment. The risk of renal failure in Godet's ${ }^{6}$ series was $25 \%$, with $8 \%$ of the patients requiring hemodialysis. Safi's ${ }^{20}$ focused analysis demonstrated a $17.5 \%$ risk of worsening renal function, with $15 \%$ requiring hemodialysis; half of the patients ultimately died. After endovascular repair of juxtarenal aneurysms, the risk for needing hemodialysis was low, but preoperative renal dysfunction was shown to adversely effect survival and increase the risk for worsening renal dysfunction. ${ }^{21}$ Similar results were suggested by the present series, although it was not statistically powered to demonstrate an association. The advantage of avoiding renal ischemia with an endovascular approach may be offset by the use of nephrotoxic contrast agents and the risk of atheroembolization due to device manipulation within the aneurysm.

Myocardial complications are infrequent but not eliminated with an endovascular approach. Patients having open thoracoabdominal aneurysm repairs at our institution undergo coronary angiography followed by revascularization, if indicated, before aneurysm repair. However, when endovascular repairs are planned, only patients with symptoms or functional studies indicative of reversible ischemia are evaluated by cardiac catheterization. In the review of Svensson et al's ${ }^{1}$ experience, an association between coronary artery disease and 30-day mortality was demonstrated. The finding that later surgical series have not shown coronary atherosclerosis to be a risk for mortality may be attributable to more aggressive screening and revascularization strategies. ${ }^{2-5}$ Whether all of the patients having endovascular 
repair of thoracoabdominal aneurysm should undergo cardiac catheterization remains to be seen.

\section{Technical Issues}

These procedures are more technically demanding than other well-established endovascular operations. The lengthy operative and fluoroscopic times coupled with large contrast doses attest to their complexity (Table 1). Experience with reconstruction techniques based upon 3-D imaging systems is critical for both patient selection and device design. Proficiency with endovascular grafting, visceral vessel disease, and the surgical skills required for conduit placement are fundamental to success. Further problem-solving skills are also required, such as the use of intravascular ultrasound, thrombolysis, and the management of distal occlusive disease. Regardless of the endovascular skill level, severe tortuosity and concomitant occlusive disease pose serious challenges to this technique as it may to the open technique. ${ }^{22}$

The endovascular approach and the design of this device place limitations on patient selection. All patients in this series had aneurysms; none had chronic dissections. Accurate device deployment and rotational movement may be limited by the small true lumen in patients having dissection. Therefore, these patients are currently treated with conventional surgery at our institution. Additional anatomic constraints on patient selection included individuals whose visceral arteries arose within approximately a $10-\mathrm{mm}$ arc length of each other. Such cases have been addressed using directional branches or hybrid approaches combined with extra-anatomic bypass, but these are more challenging.

Planning, device design, and manufacturing of custom devices create inherent delays in treatment. Therefore, patients requiring urgent or emergency repairs are relegated to open surgery. Potential for rupture in the intervening period should be considered in the overall mortality but certainly also occurs in patients awaiting elective open repair.

This technique will continue to undergo iterative improvements with respect to patient selection, device design, and delivery techniques. Adequate determination of longterm rupture protection or device durability will require longer follow-up of a stable design. Dissemination of this repair technique will also require widespread availability of adequate intraoperative imaging equipment and the mixed skill set for proper deliver. Once these issues have been addressed, a more accurate comparison of open versus endovascular treatment of thoracoabdominal aneurysms can be considered.

\section{Conclusions}

Endovascular repair of aortic aneurysms involving the visceral segment in nonsurgical candidates is feasible. The known complications of thoracoabdominal aortic aneurysm repair are not eliminated, but morbidity and mortality are low relative to the patient population studied. Further refinement of the device, technique, and patient selection is ongoing, as is assessment of durability. Future studies may include healthier patients, newer device designs, and simplified delivery systems.

\section{References}

1. Svensson LG, Crawford ES, Hess KR, Coselli JS, Safi HJ. Experience with 1509 patients undergoing thoracoabdominal aortic operations. J Vasc Surg. 1993;17:357-68

2. Coselli JS, Conklin LD, LeMaire SA. Thoracoabdominal aortic aneurysm repair: review and update of current strategies. Ann Thorac Surg. 2002;74:S1881-4.

3. Safi HJ, Miller CC III, Huynh TT, et al. Distal aortic perfusion and cerebrospinal fluid drainage for thoracoabdominal and descending thoracic aortic repair: ten years of organ protection. Ann Surg. 2003; 238:372-80.

4. Cambria RP, Clouse WD, Davison JK, Dunn PF, Corey M, Dorer D Thoracoabdominal aneurysm repair: results with 337 operations performed over a 15-year interval. Ann Surg. 2002;236:471-9.

5. Schepens M, Dossche K, Morshuis W, et al. Introduction of adjuncts and their influence on changing results in 402 consecutive thoracoabdominal aortic aneurysm repairs. Eur J Cardiothorac Surg. 2004;25:701-7.

6. Godet G, Fleron MH, Vicaut E, Zubicki A, Bertrand M, Riou B, et al. Risk factors for acute postoperative renal failure in thoracic or thoracoabdominal aortic surgery: a prospective study. Anesth Analg. 1997; $85: 1227-32$.

7. Rigberg DA, McGory ML, Zingmond DS, Maggard MA, Agustin M, Lawrence PF, et al. Thirty-day mortality statistics underestimate the risk of repair of thoracoabdominal aortic aneurysms: a statewide experience. J Vasc Surg. 2006;43:217-22; discussion 223.

8. Anderson JL, Berce M, Hartley DE. Endoluminal aortic grafting with renal and superior mesenteric artery incorporation by graft fenestration. J Endovasc Ther. 2001;8:3-15.

9. Browne TF, Hartley D, Purchas S, Rosenberg M, van Schie G, Lawrence-Brown M. A fenestrated covered suprarenal aortic stent. Eur J Vasc Endovasc Surg. 1999;18:445-9.

10. Greenberg RK, Haulon S, O'Neill S, Lyden S, Ouriel K. Primary endovascular repair of juxtarenal aneurysms with fenestrated endovascular grafting. Eur J Vasc Endovasc Surg. 2004;27:484-91.

11. Anderson JL, Adam DJ, Berce M, Hartley DE. Repair of thoracoabdominal aortic aneurysms with fenestrated and branched endovascular stent grafts. J Vasc Surg. 2005;42:600-7.

12. Greenberg RK, West K, Pfaff K, Foster J, Skender D, Haulon S, et al. Beyond the aortic bifurcation: branched endovascular grafts for thoracoabdominal and aortoiliac aneurysms. J Vasc Surg. 2006;43:87986.

13. Chuter TAM, Gordon RL, Reilly LM, Goodman JD, Messina LM. An endovascular system for thoracoabdominal aortic aneurysm repair $J$ Endovasc Ther. 2001;8:25-33.

14. O'Neill S, Greenberg RK, Resch T, Bathurst S, Fleming D, Kashyap $\mathrm{V}$, et al. An evaluation of centerline of flow measurement techniques to assess stentgraft migration in thoracic aortic stentgrafting. $J$ Vasc Surg. 2006;43:1103-10.

15. Resch T, Greenberg RK, Lyden S, Clair D, Krajewski L, Kashyap V, et al. Combined staged procedures for the treatment of thoracoabdominal aneurysms. $J$ Endovasc Ther. 2006;13:481-9.

16. Elefteriades J. Natural history of thoracic aortic aneurysms: indications for surgery, and surgical versus nonsurgical risks. Ann Thorac Surg. 2002;74:S1877-80

17. Svensson LG, Hess KR, Coselli JS, Safi HJ, Crawford ES. A prospective study of respiratory failure after high-risk surgery on the thoracoabdominal aorta. J Vasc Surg. 1991;14:271-82.

18. Black S, Wolfe J, Clark M, Hamady M, Chesire N, Jenkins M Endovascular exclusion with visceral revascularization. Vascular Annual Meeting. 2005;58:2005. 
19. Dake MD, Miller DC, Mitchell RS, Semba CP, Moore KA, Sakai T. The "first generation" of endovascular stent-grafts for patients with aneurysms of the descending thoracic aorta. $J$ Thorac Cardiovasc Surg. 1998;116:689-703; discussion 703-4.

20. Safi HJ, Harlin SA, Miller CC, Iliopoulos DC, Joshi A, Mohasci TG, et al. Predictive factors for acute renal failure in thoracic and thoracoabdominal aortic aneurysm surgery. J Vasc Surg. 1996;24: 338-44.

21. Haddad F, Greenberg RK, Walker E, Nally J, O’Neill S, Kolin G, et al. Fenestrated endovascular grafting: The renal side of the story. J Vasc Surg. 2005;1:181-90.

22. Svensson LG, Crawford ES, Hess KR, Coselli JS, Safi HJ. Thoracoabdominal aortic aneurysms associated with celiac, superior mesenteric, and renal artery occlusive disease: methods and analysis of results in 271 patients. J Vasc Surg 1992;16:378-89; discussion 389-90.

\section{Discussion}

Dr J. Coselli (Houston, Tex). Dr Roselli, congratulations on an outstanding presentation. You and your colleagues have boldly built upon a successful track record for the endovascular treatment of abdominal aortic aneurysms and a growing experience with the endovascular exclusion of juxtarenal and descending thoracic aneurysms with this innovative approach, allowing now for treatment of those aneurysms traversing the visceral aortic segment. This is indeed a significant contribution to demonstrate the feasibility of this alternative approach to the open operation from which no doubt many patients have benefited. It is notable that you have been able to demonstrate that the endovascular procedure can be carried out in many cases under regional anesthesia.

A few questions. It is a shame that this study could not have been carried out alongside controls, particularly in view of the fact that the data provided regarding the Gore TAG graft is such that at 2 years, there was no demonstrated difference in the survival rates. Additionally, I would like for you to comment on your postoperative anticoagulation regimen for these patients, and if you could, explain to us why you would not recommend the use of cerebrospinal fluid drainage in all of your patients? Because 2 of your spinal cord problems postoperatively were delayed, would you recommend the insertion of a cerebrospinal fluid catheter in such cases? And then, regarding this particular innovative technology, would you comment, based upon your experience, on the requirements for the training and qualifications for the implementation of such therapy?

Again, congratulations.

Dr Eric E. Roselli (Cleveland, Ohio). Thanks, Dr Coselli. With regard to controls, I agree, it would be nice to compare controls to these investigational patients. However, this is a technology that is still in the process of evolution, and currently we are using this selectively in patients who we don't think have a good open surgical alternative.

Our postoperative anticoagulation regimen in these patients is not based at all on the procedure, although they are anticoagulated during the procedure. Activated clotting time is maintained above 300 throughout the procedure, but then heparin is allowed to drift or is reversed with protamine in the operating room. Otherwise they receive antiplatelet therapy for concomitant coronary disease or other comorbid indications.

Cerebrospinal fluid drainage was not used in all patients. The patients that we used drainage for were at anatomic risk. So most type 1, 2, and 3 thoracoabdominal aneurysms did have drainage catheters, and also patients with type 4 aneurysms who had had previous aortic surgery-who we felt to be at an elevated risk. The 2 patients who had paraplegia did have drainage catheters in place, and unfortunately that wasn't enough to prevent them from having spinal cord ischemia. What we have learned, however, is that we may be able to predict the patients who are going to be at the highest risk based on their anatomy. Not all patients are selected for this procedure if we believe they are at too high risk for spinal cord injury. For example, a patient who has compromised internal iliac perfusion who is going to require coverage of their subclavian artery and extensive aortic coverage may not be recommended for this procedure. If those important collateral vascular beds are going to be compromised from this procedure, we will find another way to deal with them - that is, with an internal iliac branch as an additional part of the device or with a preoperative carotid subclavian bypass.

And then, with regard to the question of the requirements for training, these procedures require not only the ability to perform complicated large-bore vascular access techniques such as conduits but also other extra-anatomic bypasses such as a carotid subclavian bypass. Also, familiarity with complicated endovascular techniques, such as thrombolysis and embolization, and also the know-how to deal with all of the branches of the viscera and the aortic arch endovascularly are critical to a successful outcome.

Dr G. Wheatley (Phoenix, Ariz). Dr Roselli, I would like to compliment you on an excellent study and excellent presentation, and I would also like to compliment your colleagues and yourself on moving this field forward.

I have two questions. The first is related to the number of patients who underwent an open thoracoabdominal approach during the same period of this study, just to get a sense of how many patients were involved and whether or not the selection criteria determined who got in each study in terms of whether it was an endovascular requirement or were there other factors to determine who was selected?

My second question is, Could you elaborate a little bit more on the intense imaging in terms of $3-D$ reconstruction and mapping? I think that is one important component to the success of this procedure.

Dr Roselli. Thanks, Grayson. I don't know the exact number of patients, but I believe we have done over 100 open thoracoabdominal aneurysm repairs during this period. And you are exactly right about the importance of 3-D imaging. I didn't have time to talk about the planning of these devices, but it is very intensive. We do all the planning ourselves with the use of 3-D work stations, and it is important to be very facile with that. I should add that to the answer to Dr Coselli's second question.

Dr C. Miller (Stanford, Calif). Dr Roselli and his colleagues have shared with us some unbelievable technical wizardry today, which is most impressive. My question pertains to why an intervention was deemed necessary in these very old and very sick patients. Some of us who have been around awhile have learned the hard way in dealing with patients with thoracoabdominal aneurysms that if you tamper with the work of the Lord too often, you are going to get caught occasionally and things will not go well. You didn't tell us, Eric, or perhaps I missed it, what was the clinical indication mandating a procedure in these individuals who are old and trying to die from so many other diseases? It appears 
to me more and more that in this country one can't die with dignity anymore.

Dr Roselli. All right. Thanks, Dr Miller. You are right, these patients are sick and old; however, the indication for therapy in these patients was that they were at high risk from dying from their aneurysms. And as shown in the data, for the patients with type 1, 2 , and 3 aneurysms, the mean aneurysm size was nearly $8 \mathrm{~cm}$. We don't treat every single high-risk patient with an intervention; some patients are selected to be treated medically, but I don't have the data on the medically treated patients to make a comparison.

Dr. Miller. My question had more to do with symptoms, not just size, justifying a prophylactic operation in an asymptomatic individual. If these patients are symptomatic and presenting with pain, then sure, one probably ought to intervene; conversely, in asymptomatic very elderly patients with a large thoracoabdominal aneurysm who have multiple comorbidities and little quality of life left, I am not sure we should do anything except keep them comfortable.

Dr. Roselli. Well, that is an interesting question, but we do have over $80 \%$ survival at 1 year in these folks with large aneurysms and no late aneurysm-related deaths. To answer your question, though, no, these patients were not symptomatic. Symptomatic patients, unfortunately, are not candidates for this approach because there is an inherent delay in the design and construction of the device.

Dr S. Mitchell (Stanford, Calif). Great presentation and bold work. The question, I may have missed it, were there any left subclavian arteries covered during these repairs?

Dr Roselli. There was 1, and that patient did have a carotid subclavian bypass beforehand. 


\section{Online References}

E1. Matsumura J, Brewster D, Makaroun M, Naftel D. A multicenter controlled clinical trial of open versus endovascular treatment of abdominal aortic aneurysm. J Vasc Surg. 2003;37:262-71.

E2. Greenberg RK, Chuter TA, Sternbergh WC, III, Fearnot NE. Zenith AAA. endovascular graft: intermediate-term results of the US multicenter trial. J Vasc Surg. 2004;39:1209-18.

E3. Greenberg RK, O’Neill S, Walker E, Haddad F, Lyden SP, Svensson $\mathrm{LG}$, et al. Endovascular repair of thoracic aortic lesions with the Zenith TX1 and TX2 thoracic grafts: intermediate-term results. J Vasc Surg. 2005;1:589-96.

E4. Makaroun MS, Dillavou ED, Kee ST, Sicard G, Chaikof E, Bavaria $\mathrm{J}$, et al. Endovascular treatment of thoracic aortic aneurysms: results of the phase II multicenter trial of the GORE TAG thoracic endoprosthesis. J Vasc Surg. 2005;41:1-9.

E5. White GH, Yu W, May J, Chaufour X, Stephen MS. Endoleak as a complication of endoluminal grafting of abdominal aortic aneurysms: classification, incidence, diagnosis, and management. J Endovasc Surg. 1997;4:152-68.

TABLE E1. Complications following endovascular repair of thoracoabdominal aortic aneurysm

\begin{tabular}{lc}
\hline Morbidity & $\mathbf{n = 7 3 ( \% )}$ \\
\hline Renal & \\
$\quad$ Sustained creatinine elevation & $6(8.2)$ \\
Dialysis-dependent renal failure & $1(1.4)$ \\
Renal occlusion/stenosis & $5(6.8)$ \\
Spinal cord injury & $2(2.7)$ \\
Type II or III TAA & $2 / 28(7.1)$ \\
Respiratory failure & $5(6.8)$ \\
Tracheostomy & $2(2.7)$ \\
Myocardial infarction & $4(5.5)$ \\
Aneurysm growth & 0 \\
Rupture & 0 \\
Conversion & 0 \\
Device integrity & \\
Component separation & 0 \\
Barb fracture & 0 \\
Stent fracture & $1(2.1)$ \\
Migration & 0 \\
\hline
\end{tabular}

TAA, Thoracoabdominal aneurysm.
E6. Chaikof E, Blankensteijn JD, Harris PL, White G, Zarins C, Bernhard $\mathrm{V}$, et al. Reporting standards for endovascular aortic aneurysm repair. $J$ Vasc Surg. 2002;35:1048-60.

\section{Appendix E1: Definitions of Morbidity}

Neurologic injury included stroke and spinal cord injury and was defined by typical findings on clinical exam and confirmation by imaging and consultation with a neurologist.

Myocardial infarction was determined by rise and fall in enzymes and associated characteristics.

Respiratory failure was defined as the need for mechanical ventilation after reintubation or tracheostomy.

Renal dysfunction was defined as a sustained increase in the serum creatinine by $30 \%$ or more, loss of renal branch patency, or the need for new onset of hemodialysis. 